\title{
Delivery of Antisense Oligonucleotides Mediated by a Hydrogel System: In Vitro and In Vivo Application in the Context of Spinal Cord Injury
}

\section{Pedro M. D. Moreno1, Teresa Rodrigues,1,2, Marília Torrado1,2, Isabel F. Amaral1,2, and Ana P. Pêgo 3,4,5,6}

1- izS-Instituto de Investigação e Inovação em Saúde, Universidade do Porto, Porto, Portugal. 2-INEB-Instituto de Engenharia Biomédica, Universidade do Porto, Porto, Portugal. 3-ỉs-Instituto de Investigação e Inovação em Saúde, Universidade do Porto, Porto, Portugal. apego@ineb.up.pt.

4-INEB-Instituto de Engenharia Biomédica, Universidade do Porto, Porto, Portugal. apego@ineb.up.pt.

5-Faculdade de Engenharia da Universidade do Porto, Porto, Portugal. apego@ineb.up.pt. 6-Instituto de Ciências Biomédicas Abel Salazar (ICBAS), Universidade do Porto, Porto, Portugal. apego@ineb.up.pt.

Originally published in Methods Mol Biol. 2019;2036:205-219. doi: 10.1007/978-1-4939-9670-4_12.

\begin{abstract}
Biomaterials-based hydrogels are attractive drug-eluting vehicles in the context of RNA therapeutics, such as those utilizing antisense oligonucleotide or RNA interference based drugs, as they can potentially reduce systemic toxicity and enhance in vivo efficacy by increasing in situ concentrations. Here we describe the preparation of antisense oligonucleotide-loaded fibrin hydrogels exploring their applications in the context of the nervous system utilizing an organotypic dorsal root ganglion explant in vitro system and an in vivo model of spinal cord injury.
\end{abstract}

Key words Antisense oligonucleotides, Hydrogel, Fibrin, Gene silencing, Central nervous system, Spinal cord injury, Dorsal root ganglion

\section{INTRODUCTION}

Biomaterials-based hydrogels are three-dimensional networks with very high water content and currently under investigation for various biomedical purposes among which tissue-engineering and drug delivery show immense potential [1]. In the area of nerve regeneration, hydrogels can be seen as scaffolds able to provide not only a physical support but also a more permissive environment for 
axonal growth after injury, as well as serve as matrices to vectorize several components that can enhance the regenerative process $[2,3]$. To this regard, antisense oligonucleotides (AONs) could be promising drugs to achieve enhanced regeneration through gene expression modulation. Singlestranded modified AONs have shown the capacity to be productively, albeit unspecifically, taken up by several cell types in vitro and in vivo [4-6], including neurons in the central nervous system (CNS) [7-10], and target their cognate RNA for gene expression modulation. Thus, combination of hydrogels with AONs could provide a platform for their controlled localization and presentation to the target tissue, augmenting their activity while potentially reducing systemic toxicity concerns.

An interesting biomaterial for hydrogel formation and application in the CNS is fibrin as it has been shown to act as a permissive bridging material for axonal regeneration improving functional recovery after spinal cord injury when applied to the lesion site [11-13]. Thus, we here demonstrate the setup of an AON-loaded fibrin hydrogel platform to explore hydrogel-assisted AON delivery in two nervous system settings: the organotypic dorsal root ganglion (DRG) explant culture; and the rat spinal cord hemisection in vivo model system. Of notice is the fact that the organotypic DRG explant culture allows for the maintenance of the neuronal network and extracellular microenvironment creating an interesting and flexible in vivo-mimicking system on which to study AON-nerve cell interaction, uptake and silencing efficiency [14]. We have recently demonstrated that the fibrin-AON system provides an efficient platform for the local application of AONs inside the lesioned area of the spinal cord (in a rat spinal cord hemisection model) where AONs spread throughout the entire lesioned area achieving efficient gene silencing activity [14].

\section{Materials}

Unless mentioned otherwise all reagents are of analytical quality. Diligently follow all waste disposal regulations when disposing of waste materials.

Animal procedures need to follow ethically acceptable institutionally approved procedures and national and international regulations as well as be performed by personnel trained in laboratory animal procedures.

\subsection{Preparation and Characterization of AON-Loaded Fibrin Hydrogels}

1. Tris-buffered saline (TBS, 10 ! concentration): $1370 \mathrm{mM} \mathrm{NaCl} ; 27 \mathrm{mM} \mathrm{KCl} ; 330 \mathrm{mM}$ Tris, $\mathrm{pH}$ 7.4, in Type 2 (pure) water. Sterilize by filtration $(0.22 \mu \mathrm{m})$.

2. Fibrinogen solution: $30 \mathrm{mg} / \mathrm{mL}$ fibrinogen, 1 ! TBS $\mathrm{pH} 7.4$. Add $0.250 \mathrm{~g}$ of plasminogen-free fibrinogen from pooled human plasma containing factor XIII ( $F_{4883}$, Sigma-Aldrich) (see Note 1) to a $50 \mathrm{~mL}$ conical centrifuge tube containing $10 \mathrm{~mL}$ of Type I (ultrapure) water (resistivity of 18.3 Mega $\Omega \cdot \mathrm{cm}$ at $25^{\circ} \mathrm{C}$ ) and place it in a water bath at 37 " $\mathrm{C}$ for $4 \mathrm{~h}$ to allow solubilization, stirring gently every hour (final concentration: $25 \mathrm{mg} / \mathrm{mL}$ ). Prepare one Spectra/Por ${ }^{\circledR} 7$ standard regenerated cellulose dialysis tubing ( $8 \mathrm{kDa}$ MWCO, Spectrum ${ }^{\circledR}$ ) by previously immersing it in water for $30 \mathrm{~min}$ to remove the preservative solution, and dialyze the fibrinogen against $5 \mathrm{~L}$ of 1 ! TBS pH 7.4 at RT during $24 \mathrm{~h}$. Transfer to a conical centrifuge tube and sterilize by filtration $(0.22 \mu \mathrm{m})$ using a low protein binding filter. Determine the concentration of fibrinogen using a NanoDrop ${ }^{\mathrm{TM}} \mathrm{UV}-\mathrm{Vis}$ spectrophotometer (or alternatively, a UV-Vis spectrophotometer and a quartz cuvette) (see Note 2). 
Concentrate the fibrinogen solution in a Sartorius Vivaspin ${ }^{\circledR} 20 \mathrm{~mL}$ centrifugal concentrator ( 50 or $100 \mathrm{kDa}$ MWCO) (see Note 3 ) accordingly to the expected fibrinogen final concentration- $30 \mathrm{mg} / \mathrm{mL}$ (if dilution is needed after concentration use 1 ! TBS, $\mathrm{pH} 7.4$ ).

3. Alexa Fluor 488-labeled human fibrinogen: $(1.5 \mathrm{mg} / \mathrm{mL})$ fibrinogen from human plasma-Alexa Fluor $^{\mathrm{TM}} 488$ Conjugate (ThermoFisher Scientific, \#F13191).

4. Thrombin stock solution at 100 National Institute of Health standard (NIH) $\mathrm{U} / \mathrm{mL}$ in TBS pH 7.0. Thaw a vial with thrombin from human plasma (T7009, Sigma-Aldrich) on ice. Add slowly sterile TBS $\mathrm{pH} 7.0$ directly into the thrombin flask (use a syringe with a needle to penetrate the rubber closure on the vial) to dissolve thrombin to a final concentration of $100 \mathrm{NHI}$ units $/ \mathrm{mL}$ (see Note 4 ). Place the thrombin flask on an orbital shaker at 4 " $\mathrm{C}$ and mix at a slow speed for $2 \mathrm{~h}$. Sterilize the thrombin solution by filtration $(0.22 \mu \mathrm{m})$ using a low protein binding filter. Prepare aliquots $(25-50 \mu \mathrm{L})$ on ice (see Note 5 ) and store them at \# 20 "C. After thawed, aliquots may be stored at 4 "C for 1 week.

5. $\mathrm{CaCl}_{2}$ stock solution at $50 \mathrm{mM}$ in TBS pH 7.4. Dissolve $55.49 \mathrm{mg}$ of $\mathrm{CaCl}_{2}(102,378, \mathrm{Merck})$ in $10 \mathrm{~mL}$ of 1 ! TBS pH 7.4 and sterilize the solution by filtration $(0.22 \mu \mathrm{m})$. Prepare $200 \mu \mathrm{L}$ aliquots and store them at \# 20 "C. After thawed, aliquots may be stored at 4 "C.

6. Aprotinin stock solution at $5 \mathrm{mg} / \mathrm{mL}$ in 1 ! Phosphate buffered saline (PBS, 1!) $\mathrm{pH} 7.4$. Dissolve 10 $\mathrm{mg}$ of aprotinin ( $\mathrm{A}_{3428}$, Sigma-Aldrich) in $2 \mathrm{~mL}$ of 1 ! PBS and sterilize the solution by filtration ( 0.22 $\mu \mathrm{m}$ ) using a low protein binding filter). Prepare $20 \mu \mathrm{L}$ aliquots and store them at \# 20 " $\mathrm{C}_{i}$ after thawed, aliquots may be stored at 4 "C for 1 week.

7. AONs: $50 \mu$ MFull phosphorothioate linked, 20O-methyl-RNA single stranded oligonucleotide (Cy5*m- $C * m C * m U * m C * m U * m U * m A * m C * m C * m U * m C *-m A * m G * m U * m U * m A * m C * m A$ ) (see Note $6)$.

8. Phosphate buffered saline solution (PBS, 1!) (137 mM NaCl, $2.7 \mathrm{mM} \mathrm{KCl}, 10 \mathrm{mM} \mathrm{Na} 2 \mathrm{HPO}_{4}, 2 \mathrm{mM}$ $\mathrm{K}_{2} \mathrm{HPO}_{4}$ ).

9. Microcentrifuge tubes (o.6 mL), Maxymum Recovery (Axygen).

10. 15-well $\mu$-slide Angiogenesis (IBIDI).

11. Laser scanning confocal microscope.

\subsection{Culture of Dorsal Root Ganglion (DRG) Explants Within AON- Loaded Fibrin Hydrogels}

1. Timed pregnant (E18) 3-month-old female Wistar rats. All animals maintained under a $12-\mathrm{h} / 12-\mathrm{h}$ light/dark cycle and fed with regular rodent's chow and tap water ad libitum.

2. Hanks's balanced salt solution (GIBCO).

3. DMEM/F12-P/S medium: DMEM/F12 with GlutaMAX'M (GIBCO), 1\% (v/v) Penicillin/Streptomycin

(P/S) (Biowest). 
4. Supplemented DMEM/F-12 medium: DMEM/F12 with GlutaMAX ${ }^{\mathrm{TM}}$ (Gibco), 2\% (v/v) B-27 (Thermo Fisher Scientific), 1\% (v/v) P/S, $1.25 \mathrm{mg} / \mathrm{mL}$ amphotericin B (Capricorn Scientific), $30 \mathrm{ng} / \mathrm{mL}$ nerve growth factor (NGF) (Millipore), and $10 \mathrm{mg} / \mathrm{mL}$ aprotinin (Sigma-Aldrich).

5. Dumont 55 fine forceps.

6. Stereomicroscope with a digital camera.

7. 15-well $\mu$-Slide Angiogenesis plates (IBIDI).

8. Microcentrifuge tubes (o.6 mL), Maxymum Recovery (Axygen).

9. mirVana miRNA Isolation Kit (Ambion).

10. Standard qPCR kits (one or two-step) and equipment.

\subsection{Local Application of AON-Loaded Hydrogel in the Rat Hemisection Spinal Cord Injury Model}

1. Anesthesia machine (isoflurane-lsoVet ${ }^{\circledR}$-system).

2. Buprenorphine solution $0.3 \mathrm{mg} / \mathrm{mL}$ (Bupaq ${ }^{\circledR}$ ).

3. Ophthalmic solution (Siccafluid ${ }^{\circledR}$ ).

4. Humid food.

5. Glycosylated isotonic serum (BRAUN VET ${ }^{\circledR}$ ).

6. $\mathrm{EtOH} 70 \%(v / v)$.

7. lodopovidone solution 7.5\% (m/v) (Braunol $\left.{ }^{\circledR}\right)$.

8. Surgical equipment (scalpel, scissors, scraper, bone trimmer or rongeurs, forceps, sterile surgical compresses).

9. Digital scale.

10. Shaving machine.

11. Heating pads.

12. Materials for fibrin gel preparation as in Subheading 2.1.

13. Poly(trimethylene carbonate-co- $\varepsilon$-caprolactone) (P(TMC-CL)) patch (preparation of which is described in [15] and [16]) (see Note 7 ).

INSTITUTO DE INVESTİGAÇÃO E INOVAÇÃO EM SAUUDE UNIVERSIDADE DO PORTO 
14. RNAlater (Thermofisher).

15. 1.5 $\mathrm{mL}$ glass tissue grinder (Wheaton).

16. Standard qPCR kits (one or two-step) and equipment.

\section{Methods}

\subsection{Preparation of AON-Loaded Fibrin Hydrogels and Characterization by Confocal Microscopy}

1. Take the purified fibrinogen solution at $30 \mathrm{mg} / \mathrm{mL}$ stored at \# 80 " $\mathrm{C}$ and thaw it in a 37 "C water bath keeping it at room temperature until use. Thaw an aliquot of Alexa-Fluor-488- labeled human fibrinogen $(1.5 \mathrm{mg} / \mathrm{mL})$ on ice and keep it covered from light.

2. Thaw the aliquots containing thrombin, $\mathrm{CaCl}_{2}$, and aprotinin on ice.

3. Finally take the $\mathrm{Cy}_{5}$-labeled $\mathrm{AON}$ at initial $50 \mu \mathrm{M}$ concentration (in nuclease-free water) and place it on ice and covered from light.

4. To prepare a $20 \mu \mathrm{L} \mathrm{AON-loaded} \mathrm{fibrin} \mathrm{gel} \mathrm{drop} \mathrm{for} \mathrm{characterization,} \mathrm{the} \mathrm{fibrinogen} \mathrm{solution}$ containing $0.14 \mathrm{mg} / \mathrm{mL}$ (i.e., $1 \%$ of total amount of unlabeled fibrinogen) of Alexa Fluor 488 -labeled human fibrinogen is mixed in an $0.6 \mathrm{~mL}$ maximum recovery tube, with an equal volume of a thrombin working solution in 1 ! TBS pH 7.4 prepared from the stock solutions of thrombin, $\mathrm{CaCl}_{2}$ and aprotinin. The final concentration of fibrin components is of $14 \mathrm{mg} / \mathrm{mL}$ fibrinogen, $2 \mathrm{NIH} \mathrm{U/mL} \mathrm{human}$ thrombin, $2.5 \mathrm{mM} \mathrm{CaCl}$, and $10 \mu \mathrm{g} / \mathrm{mL}$ aprotinin.

5. Incorporate the AONs (at $6 \mu \mathrm{M}$ final concentration) into the fibrinogen solution before mixing with the thrombin working solution. Homogenize the polymerizing solution with the help of a $10 \mu \mathrm{L}$ micropipette by pipetting three times up and down before transferring it to 15 -well $\mu$-slide Angiogenesis. Readily incubate it for $30 \mathrm{~min}$ at 37 " $\mathrm{C}$ in a $5 \% \mathrm{CO}_{2}$ humidified incubator to allow fibrin cross-linking by factor XIIla.

6. Before imaging in a laser scanning confocal microscope, check gelation visually and hydrate the gel with $40 \mu \mathrm{L}$ PBS.

7. AONs were previously observed to integrate into the fibrin network being closely colocalized with the fibrin fibers and not freely diffusing in the aqueous portion of the gel, while also leading to an increase in the average pore area of the fibrin network (Fig. 1) (see Note 8 ). 
A.

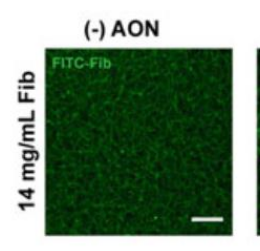

$$
\text { (+) AON }
$$

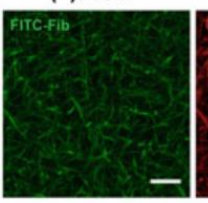

$(+)$ AON

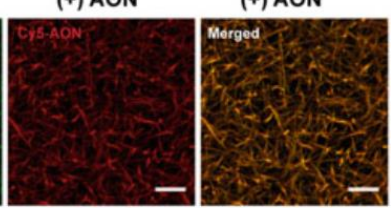

B.

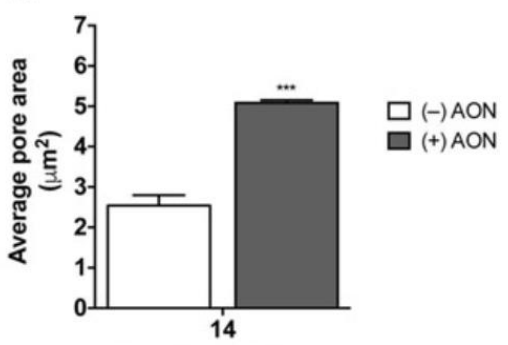

[Fibrinogen] $\mathrm{mg} / \mathrm{mL}$

Fig. 1 Characterization of fluorescently labeled fibrin hydrogels using confocal microscopy. (a) Representative maximum Z-projections of confocal stack images of the corresponding fibrin gels (at fibrin concentrations of $14 \mathrm{mg} / \mathrm{mL}$ containing $1 \%$ (w/w) FITC- Fibrinogen). Complete association (colocalization) of Cy5-AONs with fibrin fibers (FITC-Fib) is observed. Scale bars, $10 \mu \mathrm{m}$. (b) Analysis of average pore area using confocal microscopy images taken from the fibrin gels. Maximum Zprojections were used to calculate the average pore area per image field using MATLAB. Two-way ANOVA, followed by Bonferroni post hoc test, was used for statistical analysis (mean $\$$ standard deviation, $\mathrm{n} 1 / 43$ image fields per gel; $* * * * \mathrm{p}<0.0001 ; * * * \mathrm{p} 1 / 40.0001$ ). Adapted from [14] and used with permission from the American Society of Gene \& Cell Therapy

\subsection{Gene Silencing in Dorsal Root Ganglion (DRG) Explants Mediated by AON-Loaded Fibrin Hydrogels}

\subsubsection{Rat DRG Explant Isolation}

1. Sacrifice a pregnant adult Wistar rat (E18, considering E0.5 at the morning when a vaginal plug is detected) by $\mathrm{CO}_{2}$ asphyxiation. Place the animal in the induction chamber set to 7 min of induction and 2 min of dwell time. To assure death, do an incision in the jugular vein. Confirm death by absence of heart beat.

2. Harvest embryos by caesarean section. For that, disinfect all the abdomen with $70 \%(\mathrm{v} / \mathrm{v})$ ethanol and perform a C-cut in the skin, along the inguinal area. Then, to avoid damage of the uterus, lift the abdominal wall and cut through in the same manner. Gently pull the gravid uterus and collect it by cutting the uterine veins and arteries and separating it from the vagina.

3. Place the entire uterus inside a falcon containing sterile ice-cooled HBSS and leave it on ice for at least $10 \mathrm{~min}$ in order to anesthetize embryos.

4. Carefully, open the uterus wall with a scissors and collect all embryos to a petri dish with the same cold medium.

5. Under a stereomicroscope, sacrifice embryos by decapitation. Place the remaining body in their prone position in a petri dish containing DMEM/F12-P/S medium.

6. Separate the tail from the body and do an incision throughout the midline of the spinal column using fine forceps.

INSTITUTO DE INVESTİGAÇÃO E INOVAÇÃO EM SAÚDE UNIVERSIDADE DO PORTO

Rua Alfredo Allen, 208 4200-135 Porto Portugal +351220408800 
7. Gently pull away the spinal cord, leaving the DRGs exposed. At E18 the spinal cord should separate easily, leaving the DRGs in their cavities.

8. Finally, with Dumont 55 fine forceps, collect DRGs with extremely care to maintain their entire structure and conserve them in a petri dish with ice-cold DMEM/F12-P/S medium until further use (see Note 9 ).

\subsubsection{Embedding and Culture of DRGs in Hydrogel}

1. Inside a laminar flow chamber for sterile conditions and with the help of a stereomicroscope with a digital camera, plate a DRG explant in the center of a well of a 15-well $\mu$-Slide Angiogenesis plate, using Dumont 55 fine forceps (see Note 10).

2. Prepare $20 \mu \mathrm{L}$ fibrin gels as described in Subheading 3.1 except:

(a) No fluorescently labeled fibrinogen is used.

(b) A $6 \mu \mathrm{M}$ final concentration of AON (LNA-based, fully phosphorothioated gapmers) against the gene target of interest is used (in our previous work [14] an LNA-AON gapmer (LNA 6624) was used to down-regulate GSK3 $b:+C *+A *+T * g * a * t * c * t * g * g * a *+G *+C *+T$, and as control an LNA-AON

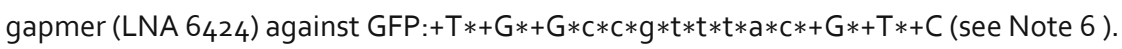

Each mixture should be done just before plating, as it polymerizes fast.

3. Quickly, add the polymerizing fibrin gel to the previous platted DRG, making sure that it stays as centered as possible. After a few seconds the gel should turn opaque indicating that fibrinogen polymerization has started.

4. Repeat the same procedure with the other wells and place the plate in a 37 " $\mathrm{C}, 5 \% \mathrm{CO}_{2}$ humidified incubator for 30 min to allow fibrin cross-clinking by factor XIIla.

5. After that time, add $40 \mu \mathrm{L}$ of supplemented DMEM/F-12 medium to each well and place it back in the incubator.

6. Culture DRG explants for up to 7 days, changing the medium every 3 days of culture. For that, remove half volume of the medium in each well and add new supplemented DMEM/F- 12 medium.

7. After 7 days of culture, collect DRGs to Maxymum recovery microcentrifuge tubes $(0.6 \mathrm{~mL})$ containing $300 \mu \mathrm{L}$ of lysis solution of the mirVana miRNA Isolation Kit and preserve it at \# 80 "C until analysis (see Note 11).

8. Proceed with RNA isolation following the manufacturer's protocol. After, a one-step qPCR kit can be used for quantitative real-time PCR for gene expression analysis. An example result is shown in Fig. 2. 

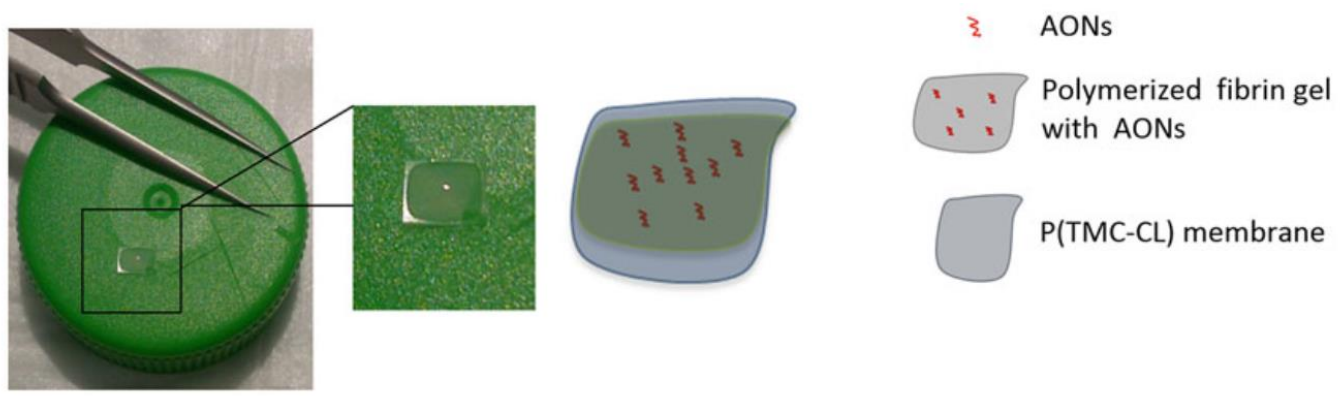

Fig. 2 View of PTMC-CL polymer patch with a drop of AON-containing gel formed on top. The patch is transferred to the lesion site with the gel side contacting the spinal cord. The schematic drawing shows the system as it is transferred to the lesion site, composed by the PTMC-CL membrane patch, then below the prepolymerized fibrin gel containing the AONs which will contact the lesion

\subsection{Gene Silencing by Local Application of AON-Loaded Hydrogel in the Rat Hemisection Spinal Cord Injury Model}

\subsubsection{Application of AON-Loaded Hydrogel After Spinal Cord Hemisection}

First of all, the animals are handled to accustom them to experimental manipulation and recognize the experimenter, in order to minimize stress. After animals' habituation, the surgery is performed and for its success the operating environment is cleaned, the instruments are sterilized, the animal is prepared (asepsis) and lastly the surgeon is prepared.

1. Weigh the animal.

2. Anaesthetize the animal with isoflurane, with induction rate of $5 \%$ and maintenance rate ranging between $1.5 \%$ and $3 \%$ (see Note 12 ).

3. Shave the animal fur from T10 vertebra until the ears and clean: 3 times with $\mathrm{EtOH} 70 \%(\mathrm{v} / \mathrm{v})$ and iodopovidone solution intercalated.

4. Inject buprenorphine solution and glycosylated serum (3 $\mathrm{mL}$ ) subcutaneously.

5. Perform a single incision between T10 vertebrae and approximately above the shoulder blade. Insert the retractor. Cut the fat (scissors), remove the muscle (scraper) and cut the muscle right above the T7-T9 vertebrae. Clean the vertebrae $T_{7}$ and $T_{8}$ using the scraper. Cut the bone (bone trimmer or rongeur) right under the vertebrae T8 and remove the vertebrae (laminectomy). Spinal cord will be immediately visible.

6. With the microscissors cut the spinal cord dorsally, approximately $2-3 \mathrm{~mm}$ in depth with one single cut (see Note 13 ). 
7. Using the procedures for hydrogel preparation as in Subheading 3.1 (except here, to achieve a faster gelation time, $25 \mathrm{U} / \mathrm{mL}$ of final thrombin concentration and $25 \mu \mathrm{g} / \mathrm{mL}$ of final aprotinin concentration) are used for in vivo experimentation as well as AON at an initial concentration of 5 $\mathrm{mM})$ the gel $(5 \mu \mathrm{L})$ is prepared inside a clean maximum recovery $0.6 \mathrm{~mL}$

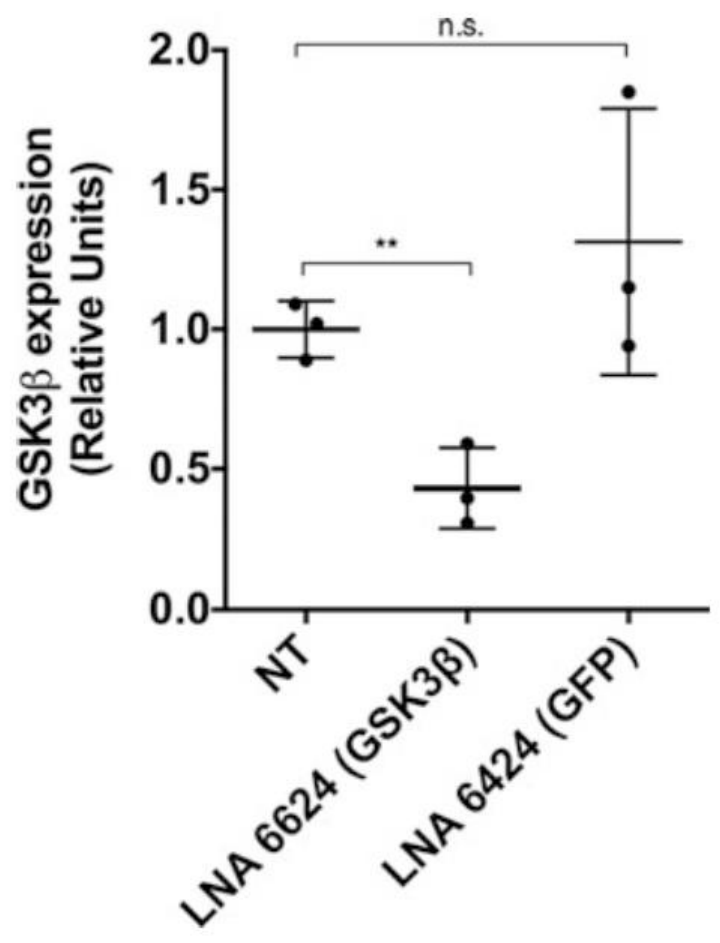

Fig. 3 Downregulation of $\mathrm{GSK}_{3} \beta$ in DRG explants mediated by an AON-loaded fibrin hydrogel. Relative RNA quantification by qRT-PCR after a 7-day exposure to LNA AONs. LNA AONs against an irrelevant sequence (LNA6424-GFP) were used as controls. Each point represents an independent experiment where RNA from a pool of 10-15 independently treated DRG explants was used per condition and per independent experiment for qPCR quantification. Results indicate mean \$SD. One way-ANOVA with Dunnett's multiple comparison test (versus nontreated [NT]) was used where indicated (*p $<0.05 ; * *<<0.01 ;$ n.S., not significant). Adapted from [14] and used with permission from the American Society of Gene \& Cell Therapy

microtube. After mixing both component mixtures, the gel ( $5 \mu \mathrm{L}$, containing $5 \mathrm{nmol}$ of $\mathrm{AON}$ ) is quickly transferred to the top of a PTMC-CL patch of around $0.5 \mathrm{~cm}$ length and $0.3 \mathrm{~cm}$ wide (methods for the preparation of pTMC-CL fiber patch are reported elsewhere in [15] and [16]) and set it aside to polymerize (Fig. 3).

A second $5 \mu \mathrm{L}$ drop is then made in another tube and pipetted into the lesioned site of the spinal cord just as it starts to turn into gel (it should take less than $1 \mathrm{~min}$ ) (see Note 14 ). Let stabilize for $1 \mathrm{~min}$ and then transfer the patch with the initial $5 \mu \mathrm{L}$ drop onto the top of the lesion such that the gel drop is underneath the patch and in contact with the lesion (this gives a total amount of $10 \mathrm{nmol}$ of AONs 
applied to the lesion site, however other AON quantities can be tested). A schematic picture of the test system is shown in Fig. 4 a.

8. Suture the musculature and subcutaneous tissue closed and lastly suture the skin, using PGA (polyglycolic acid) surgical suture thread (see Note 15 ).

A.

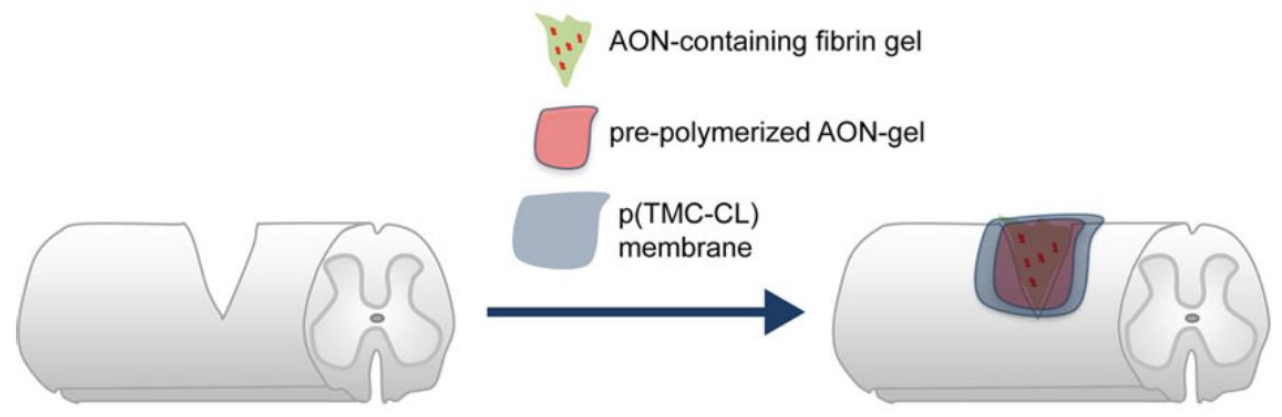

B.

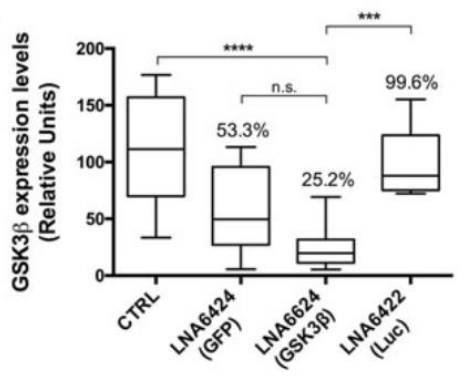

Fig. 4 Overview of the gene silencing hydrogel system locally applied to the injured spinal cord (rat spinal cord hemisection model system). (a) Schematic representation of the AON-hydrogel system for local downregulation of gene targets in the lesioned spinal cord. (b) Functional activity of LNA AONs after local application of the AON-loaded fibrin gel delivery system, evaluated 5 days postlesion. Fibrin gel with no AONs was applied in the lesion of control group rats (CTRL). LNA AONs against GFP or Luciferase (LNA6424-GFP and LNA6422-LuC) were also used as additional controls. Relative quantification of GSK3 $\beta$ RNA levels by $q R T-P C R$ is indicated. Values above box plots refer to $\mathrm{GSK}_{3} \mathrm{~b}$ expression levels as relative mean percentages. Error bars represent minimum-maximum (min-max), with line at median (control, n 1/4 9; LNA6624, n1/411; LNA6424, n1/411; LNA6422, n 1/4 7). One-way ANOVA, with Tukey multiple comparison, was used for statistical analysis ( $* * * p<0.001$, $* * * * p<0.0001$; n.S., not significant). Adapted from [14] and used with permission from the American Society of Gene \& Cell Therapy

\subsubsection{Spinal Cord Extraction for Gene Expression Analysis}

1. Five days post- $\mathrm{SCl}$, euthanize the animals using a $\mathrm{CO}_{2}$ chamber (to assure death, cut the first spinal cord segments, immediately caudal to the occipital bone). 
2. Sprinkle the animal fur with $\mathrm{EtOH} 70 \%(\mathrm{v} / \mathrm{v})$ and using a tissue scissors, cut the skin over the vertebral column from the hip to the head.

3. Using a bone scissors, cut the vertebrae dorsally and delimit your region of interest. After that, cut muscle and bone, laterally in both sides along the vertebral column. At this point, the vertebral column should be separated from the body.

4. Gently remove each vertebra, cutting with a bone trimmer or rongeurs in both sides laterally to the dorsal lamina. The vertebral canal is open and the spinal cord exposed.

5. Using a tissue scissors, cut the nerve roots along the spinal cord segments and carefully remove the spinal cord from the ventral bone using a scraper.

6. Spinal cord is then put into a centrifuge tube with chilled RNAlater in a volume enough to cover it entirely (see Note 16 ).

7. The portion of the spinal cord including the lesion (around $0.5 \mathrm{~cm}$ to each side centered at the lesion) is cut with a clean scalpel and weighed.

8. Tissue is then chopped into smaller pieces for RNA extraction using miRvana miRNA Isolation Kit. A $1 \mathrm{~mL}$ tissue grinder on ice was used for tissue homogenization before proceeding with remaining of RNA extraction protocol following the manufacturer's protocol.

9. Quantitative RT-PCR can then be used for analysis of gene silencing results (Fig. 4b).

\section{Notes}

1. Fibrinogen (as received) is stored at \# 20 "C. Prior to weighing fibrinogen, allow it to equilibrate at RT.

2. To determine the concentration of fibrinogen:

(a) Dilute fibrinogen solution 1:40 in 1! TBS 7.4 in a microcentrifuge tube; prepare 3 replicates;

(b) At the NanoDrop ${ }^{\mathrm{TM}}$, apply $2 \mu \mathrm{L}$ of 1 ! TBS 7.4 onto the measurement pedestal to measure the blank; pipette an aliquot $(2 \mu \mathrm{L})$ of each sample onto the pedestal and measure the absorbance at $280 \mathrm{~nm}$ and at $320 \mathrm{~nm}$;

(c) Determine fibrinogen concentration $(\mathrm{mg} / \mathrm{mL})$ according to the following equation, where $\varepsilon$ corresponds to the mass extinction coefficient of a solution of $1 \mathrm{mg} / \mathrm{mL}$ of fibrinogen at $280 \mathrm{~nm}(1.51$ $\mathrm{L} \mathrm{g \#} 1 \mathrm{~cm} \# 1$ ); calculate the mean and multiply by the dilution factor (40) to obtain the concentration of the purified fibrinogen solution.

$$
\frac{\left(A_{280}-A_{320}\right)}{\varepsilon}=[\text { Fibrinogen }]
$$


3. For concentration of fibrinogen, first sterilize the body of the Vivaspin ${ }^{\circledR}$ device with $70 \%(\mathrm{v} / \mathrm{v})$ ethanol; incubate for $20 \mathrm{~min}$, centrifuge at 3000 ! g for $2 \mathrm{~min}$ and discard the ethanol; repeat the procedure twice with sterile water to remove any traces of ethanol; Transfer the fibrinogen solution to the concentrator and centrifuge at 3750 ! g for $15 \mathrm{~min}$ at RT until reaching the desired volume; Check again the concentration of fibrinogen in the NanoDrop and if needed adjust the concentration to $30 \mathrm{mg} / \mathrm{mL}$ with sterile 1 ! TBS pH 7.4 in the LFC; Prepare $200 \mu \mathrm{L}$ aliquots and store them at \# 80 "C. After thawed, aliquots may be stored at 4 "C for 1 week

4. Thrombin is diluted in 1! TBS $\mathrm{pH} 7.0$ since thrombin activity is reduced

5. Thrombin aliquots are prepared in microcentrifuge tubes previously coated with $0.1 \%(\mathrm{w} / \mathrm{v})$ bovine serum albumin (BSA). For BSA coating, weight $100 \mathrm{mg}$ of BSA (Albumin fraction $\mathrm{V}, 1.12018 .0025$, Merck) and transfer it to a conical centrifuge tube containing $10 \mathrm{~mL}$ of 1 ! TBS pH 7.0. Sterilize $1 \%(\mathrm{w} / \mathrm{v})$ BSA by filtration $(0.22 \mu \mathrm{m})$ with a low protein binding filter into a new conical centrifuge tube and heat inactivate it at 70 "C for $5 \mathrm{~min}$ ( $1 \% \mathrm{w} / \mathrm{v}$ BSA stock solution may be stored at \# 20 "C). Dilute $1 \%$ $(w / v)$ BSA stock solution with sterile 1 ! TBS pH 7.0 to obtain $0.1 \%$ (w/v) BSA. Pipette $100 \mu \mathrm{L}$ of $0.1 \%$ (w/v) BSA into $1.5 \mathrm{~mL}$ microcentrifuge tubes and incubate them at 37 " $\mathrm{C}$ for $1 \mathrm{~h}$ in orbital shaker at $100 \mathrm{rpm}$. Carefully aspirate the BSA solution before use.

6. DNA bases are written in small letters; 20-O-methyl RNA bases are written as $\mathrm{mN}(\mathrm{N}$, nucleotide), phosphorothioate linkages are indicated by an asterisk. Oligonucleotides should always be HPLC purified. Several oligonucleotide synthesis companies are available for ordering such oligonucleotides. When received, oligonucleotides (lyophilized) are diluted in nuclease-free water (non-DEPC treated, Qiagen) aliquoted and stored at \# 20 "C.

7. The poly(trimethylene carbonate-co- $\varepsilon$-caprolactone) ( $(\mathrm{P}(\mathrm{MC}-\mathrm{CL}))$ patch does not influence the fibrin inserted in the lesion site, however it can be used to further increase the amount of AONs being released into the lesion as it serves as a barrier for outward diffusion by helping to contain and isolate the lesion site.

8. To measure the pore size area of fibrin gel the following specifications are recommended when acquiring the photos: use a Plan-Apochromat !63/1.4NA Oil objective and acquire images of ten optical sections covering a depth of $10 \mu \mathrm{m}$. In MatLab software, and using a 2D projection of the previously taken optical sections, pores can then be segmented and areas computed.

9. Despite being a time-consuming procedure, DRG isolation should be done as quickly as possible to maintain the explants integrity and cell viability.

10. To help transferring the DRG to the center of the well, add a drop of medium (approx. $2 \mu \mathrm{L}$ ) before plating it.

11. With the mirVana RNA extraction kit around ten DRGs need to be pulled together for RNA extraction in order to have good quality RNA at enough amounts for subsequent analysis. However, other methods of RNA isolation can in principle be used if RNA from single DRGs is desirable.

12. To guarantee that animals are fully anaesthetized, postural, tail and paw reflexes are analyzed and procedures are only carried out when no response from the animals is observed (e.g., loss of 
posture and absence of tail pinch and pedal withdrawal reflexes). Use an ophthalmic solution (SiccaFluid) in order to avoid injuries of cornea and electrical heating pads to maintain body temperature during surgery and throughout the recovery period.

13. Control any bleeding that appears after the lesion using cotton swabs or hemostatic gauze to make sure the gel is transferred only when bleeding has stopped.

14. To help identifying when the gel is being formed adjust the pipette with a volume higher than the volume you are transferring. Then keep gently pushing the pipette piston up and down just enough that you see the gel solution descending to the tip. You should notice a higher resistance to the pipetting of the solution building up. Just when you start noticing this effect immediately transfer the "about to" polymerize gel into the lesion site. Take care not to push too much the pipette tip into the lesion site.

15. Make available humid/soft food and reestablish water access after surgery. It is important the frequent bladder management (empty the animals' bladder twice a day and immediately after the surgery) and maintain the animals on a heat pad during $24 \mathrm{~h}$ post injury. Also administer buprenorphine for 3 days (twice a day, subcutaneously). Assess the animals' physical appearance and wounds daily, as well as record their weight variations. In case problems emerge with the health status of the animal evaluate possible humane ending point (euthanasia).

16. The extracted spinal cord can be stored in RNAlater at 4 " $\mathrm{C}$ overnight before continuing with the RNA extraction protocol or before freezing at \# 80 "C for long-term storage.

\section{Acknowledgements}

This work was supported by Fundação para a Ciência e a Tecnologia (FCT, Portugal) in the framework of the Harvard-Portugal. Medical School Program [HMSP-ICT/0020/2010]; Project NORTE-01-0145FEDER-000008, supported by Norte Portugal Regional Operational Programme (NORTE 2020), under the PORTUGAL 2020 Partnership Agreement, through the European Regional Development Fund (ERDF), Fundo Europeu de Desenvolvimento Regional funds through the COMPETE 2020Operacional Program for Competitiveness and Internationalization (POCl), Portugal 2020; by Portuguese funds through FCT/Ministério da Ciência, Tecnologia e Ensino Superior in the framework of the project "Institute for Research and Innovation in Health Sciences" (POCl-01-0145-FEDER007274); Santa Casa da Misericordia de Lisboa-Prémio Neurociências Mello e Castro (MC-10682015) and the fellowships SFRH/BPD/108738/ 2015 (FCT) to P.M.D.M and Infarmed (FIS-FIS-201501_CCV_20150630-88) to M.T.

\section{REFERENCES}

1. Chai Q, Jiao Y, YuX (2017) Hydrogels for biomedical applications: their characteristics and the mechanisms behind them. Gels 3:6-15. https://doi.org/10.3390/ gels3010006 
2. Pêgo AP, Kubinova S, Cizkova D et al (2012) Regenerative medicine for the treatment of spinal cord injury: more than just promises? J Cell Mol Med 16:2564-2582. https://doi. org/10.1111/j.1582-4934.2012.01603.x

3. Carballo-Molina OA, Velasco I (2015) Hydrogels as scaffolds and delivery systems to enhance axonal regeneration after injuries. Front Cell Neurosci 9:13. https://doi.org/ 10.3389/fncel.2015.00013

4. Stein CA, Hansen JB, Lai J et al (2010) Efficient gene silencing by delivery of locked nucleic acid antisense oligonucleotides, unassisted by transfection reagents. Nucleic Acids Res 38:e3. https://doi.org/10.1093/nar/

gkp841

5. Straarup EM, Fisker N, Hedtj€arn M et al (2010) Short locked nucleic acid antisense oligonucleotides potently reduce apolipoprotein B mRNA and serum cholesterol in mice and non-human primates. Nucleic Acids Res 38:7100-7111. https://doi.org/10.1093/ nar/gkq457

6. Crooke ST, Wang S, Vickers TA et al (2017) Cellular uptake and trafficking of antisense oligonucleotides. Nat Biotechnol 35:230-237. https://doi.org/10.1038/nbt.3779

7. Passini MA, Bu J, Richards AM et al (2011) Antisense oligonucleotides delivered to the mouse CNS ameliorate symptoms of severe spinal muscular atrophy. Sci Transl Med 3:72ra18. https://doi.org/10.1126/ scitranslmed.3001777

8. Kordasiewicz HB, Stanek LM, Wancewicz EV et al (2012) Sustained therapeutic reversal of Huntington's disease by transient repression of Huntingtin synthesis. Neuron 74:1031-1044. https://doi.org/10.1016/j.neuron.2012.05. 009

9. Khorkova O, Wahlestedt C (2017) Oligonucleotide therapies for disorders of the nervous system. Nat Biotechnol 35:249-263. https:// doi.org/10.1038/nbt.3784

10. Smith CIE, Zain R (2018) Therapeutic oligonucleotides: state of the art. Annu Rev Pharmacol Toxicol. https://doi.org/10.1146/ annurev-pharmtox-010818-021050

11. Johnson PJ, Parker SR, Sakiyama-Elbert SE (2009) Controlled release of neurotrophin-3 from fibrin-based tissue engineering scaffolds enhances neural fiber sprouting following subacute spinal cord injury. Biotechnol Bioeng 104:1207-1214. https://doi.org/10.1002/ bit.22476

12. King VR, Alovskaya A, Wei DYT et al (2010) The use of injectable forms of fibrin and fibronectin to support axonal ingrowth after spinal cord injury. Biomaterials 31:4447-4456. https://doi.org/10.1016/j.biomaterials. 2010.02.018

13. Sharp KG, Yee KM, Steward O (2014) A re-assessment of long distance growth and connectivity of neural stem cells after severe spinal cord injury. Exp Neurol 257:186-204. https://doi.org/10.1016/j.expneurol.2014. 04.008

14. Moreno PMD, Ferreira AR, Salvador D et al (2018) Hydrogel-assisted antisense LNA Gapmer delivery for in situ gene silencing in spinal cord injury. Mol Ther Nucleic Acids

11:393-406. https://doi.org/10.1016/j.

omtn.2018.03.009

15. Pires LR, Rocha DN, Ambrosio L, Pêgo AP (2015) The role of the surface on microglia function: implications for central nervous system tissue engineering. J R Soc Interface 12:20141224-20141224.

https://doi.org/10.1098/rsif.2014.1224

16. Pires LR, Lopes CDF, Salvador D et al (2017) Ibuprofen-loaded fibrous patches-taming inhibition at the spinal cord injury site. J Mater Sci Mater Med 28:157. https://doi.org/10. 1007/s10856-017-5967-7 